VOL. 43 (1991) [251-256]

\title{
PROJECTIVE SUBMERSIONS
}

Fabio Podestà

\begin{abstract}
We consider $C^{\infty}$ manifolds endowed with torsionfree affine connections and $C^{\infty}$ projective submersions between them which, by definition, map geodesics into geodesics up to parametrisation. After giving a differential characterisation of these mappings, we deal with the case when one of the given connections is projectively flat or satisfies certain conditions concerning its Ricci tensor; under these hypotheses we prove that the projective submersion is actually a covering.
\end{abstract}

\section{Preliminaries}

We shall recall here only some basic notions and we refer to [3] for a more detailed exposition.

Two torsionfree affine connections $\nabla^{1}$ and $\nabla^{2}$ on a $C^{\infty}$ n-dimensional manifold $M$ will be said to be projectively equivalent if they define the same system of geodesics up to parametrisation. It is easy to see that $\nabla^{1}$ and $\nabla^{2}$ are equivalent if and only if there exists a 1 -form $\sigma$ such that

$$
\nabla_{X}^{1} Y-\nabla_{X}^{2} Y=\sigma(X) Y+\sigma(Y) X
$$

for all vector fields $X, Y$ on $M$. The equivalence class of a torsionfree affine connection is said to be a projective structure $\mathcal{P}$ on $M$. To each projective structure $\mathcal{P}$ we can associate a $(1,3)$ tensor, called the Weyl projective curvature tensor $W$, which measures the projective flatness; namely $W$ vanishes if and only if we can find a local diffeomorphism onto an open set of $\mathbf{R P}^{\boldsymbol{n}}$ carrying geodesics into geodesics of $\mathbf{R P}^{n}$ relative to its standard metric.

To give a simplified expression for the Weyl tensor, we note (see [3]) that each connection belonging to a given projective structure $\mathcal{P}$ is projectively equivalent to an affine connection with symmetric Ricci tensor; if $\nabla$ is a connection belonging to $\mathcal{P}$ with symmetric Ricci tensor $S$ and curvature tensor $R$, then the Weyl curvature tensor can be expressed in the following way

$$
W(X, Y) Z=R(X, Y) Z+\frac{1}{n-1}[S(X, Z) Y-S(Y, Z) X]
$$

for all vector fields $X, Y, Z$.

Received 12 April 1990

Copyright Clearance Centre, Inc. Serial-fee code: 0004-9729/91 \$A2.00+0.00. 


\section{Projective submersions}

Throughout the following $M, N$ will denote $C^{\infty}$-manifolds endowed with projective structures $\mathcal{P}_{M}, \mathcal{P}_{N}$.

Definition 1.1: A $C^{\infty}$ submersion $f: M \rightarrow N$ is said to be projective if $f$ maps geodesics into geodesics up to parametrisation.

Har'el in [5] has proved the following theorem for which we prefer to give another proof.

TheOREM 1.1. A $C^{\infty}$ submersion $f: M \rightarrow N$ is projective if and only if, given two linear torsionfree connections $\nabla \in \mathcal{P}_{M}$ and $D \in \mathcal{P}_{N}$, there is a 1-form $\sigma \in \Omega^{1}(M)$ such that

$$
D_{X} f_{*} Y-f_{*}\left(\nabla_{X} Y\right)=\sigma(X) f_{*}(Y)+\sigma(Y) f_{*}(X)
$$

for all vector fields $X, Y$ on $M$.

ProOF: In one direction it is clear. For the converse we first denote by $\bar{\nabla} f_{*}$ the covariant derivative of $f_{*}$ as a section of the bundle $T M^{*} \otimes f^{-1} T N$ with respect to the connections $\nabla$ and $D$; moreover we denote by $K$ the distribution given by Ker $f_{*}$. We pick a point $x_{0} \in M$ and note that for all $X \in T M_{x_{0}}-K$ there is a real number $\sigma(X)$ such that

$$
\bar{\nabla}_{X} f_{*} X=2 \sigma(X) f_{*}(X)
$$

at $x_{o}$, simply because $f$ maps geodesics into geodesics. So we have defined a mapping $\sigma: T M_{x_{0}}-K \rightarrow \mathbf{R}$ by means of (1.1).

Lemma 1. If $X, Y \in T M_{x_{0}}-K$ and $X+Y \in T M_{x_{0}}-K$, then

$$
\sigma(X+Y)=\sigma(X)+\sigma(Y)
$$

Proof: We have to consider two cases:

(1) $X-Y=V \in K$. Then since $\left(\nabla_{V} f_{*}\right) V=0$, we have by linearity

$$
\begin{aligned}
& 8 \sigma(X) f_{*}(X)=4 \sigma(X) f_{*}(X)+4 \sigma(Y) f_{*}(Y) \\
& =2\left(\nabla_{X+Y} f_{*}\right)(X+Y)+\left(\nabla_{X-Y} f_{*}\right)(X-Y)=4 \sigma(X+Y) f_{*} X
\end{aligned}
$$

hence $\sigma(X+Y)=2 \sigma(X)=2 \sigma(Y)=\sigma(X)+\sigma(Y)$ and we are done.

(2) $X-Y \notin K$ and the thesis follows by polarization.

Now we want to extend the definition of $\sigma$ to $K$ : we pick $X \in T M_{x_{o}}-K$ and $V \in K$; we define

$$
\sigma(V)=\sigma(X+V)-\sigma(X)
$$


and we have to verify that this definition does not depend on the choice of $X$. So let $Y \in T M_{x_{0}}-K$ and distinguish two cases again:

(1) $X-Y=V \in K$. Then since $\left(\nabla_{W} f_{*}\right)(V)=0$ for all $W \in K$, we have

$$
\begin{aligned}
& (\sigma(X+V)-\sigma(X)) f_{*}(X)=\left(\nabla_{X} f_{*}\right)(V) \\
& =\left(\nabla_{Y} f_{*}\right)(V)=(\sigma(Y+V)-\sigma(Y)) f_{*}(Y)
\end{aligned}
$$

and we are done.

(2) $X-Y \notin K$. Then since $\left(\nabla_{X} f_{*}\right)(V)=\left(\nabla_{X-Y} f_{*}\right)(V)+\left(\nabla_{Y} f_{*}\right)(V)$ we get,

$$
\begin{gathered}
(\sigma(X+V)-\sigma(X)) f_{*} X \\
=(\sigma(X-Y+V)-\sigma(X-Y)) f_{*}(X-Y)+(\sigma(Y+V)-\sigma(Y)) f_{*}(Y),
\end{gathered}
$$

and since $f_{*}(X)$ and $f_{*}(Y)$ are linearly independent, we have

$$
\sigma(Y+V)-\sigma(Y)=\sigma(X-Y+V)-\sigma(X-Y) .
$$

But since $X+V-Y \notin K$, by Lemma 1 we have that $\sigma(X-Y)=\sigma(X)-\sigma(Y)$ and $\sigma(X-Y+V)-\sigma(X-Y)=\sigma(X+V)-\sigma(X)$; if we use this in (1.2), we get our thesis. The linearity of the extended map $\sigma$ on $T M_{x_{0}}$ is left to the reader.

Example: We consider the product manifold $M \times \mathrm{R}$ and we denote by $V$ the standard vector field $\partial / \partial t$ on $\mathbf{R}$. We construct on $M \times \mathbf{R}$ the torsionfree linear connection $\tilde{\nabla}$ given by

$$
\begin{gathered}
\tilde{\nabla}_{X} Y=\nabla_{X} Y+\gamma(X, Y) V \\
\tilde{\nabla}_{X} V=X
\end{gathered}
$$

where $X, Y$ are vector fields on $M$ and $\gamma(X, Y)=S(X, Y) /(m-1)$, with $m=\operatorname{dim} M$ and $S$ the symmetrised Ricci tensor of $\nabla$. It is very easy to verify that the projection $\pi: M \times \mathbf{R} \rightarrow M$ is projective and that $\sigma(V)=1, \sigma(X)=0$ for every vector field $X$ on $M$.

A lengthy but straightforward calculation shows that the curvature tensors $R^{\nabla}$ and $R^{D}$ are related by the following equation

$$
R^{D}\left(f_{*} X, f_{*} Y\right) f_{*} Z=f_{*}\left\{R^{\nabla}(X, Y) Z+2 d \sigma(X, Y) Z+\Sigma(X, Z) Y-\Sigma(Y, Z) X\right\}
$$

where we have put $\Sigma(X, Y)=\nabla_{X} \sigma(Y)-\sigma(X) \sigma(Y)$.

Proposition 1.1. If $\mathcal{P}_{M}$ is flat, so is $\mathcal{P}_{N}$.

Proof: We know (see [3]) that we can choose $\nabla$ and $D$ with symmetric Ricci tensors. We put $\gamma=\operatorname{Ric}^{\nabla} /(m-1)$, where $m=\operatorname{dim} M$; by assumption

$$
R^{\nabla}(X, Y) Z=\gamma(Y, Z) X-\gamma(X, Z) Y .
$$


If we make use of this in (1.3), we get

$$
\begin{aligned}
R^{D}\left(f_{*} X, f_{*} Y\right) f_{*} Z= & (-\gamma(X, Z)+\Sigma(X, Z)) f_{*} Y-(-\gamma(Y, Z)+\Sigma(Y, Z)) f_{*} X \\
& +2 d \sigma(X, Y) f_{*} Z
\end{aligned}
$$

We now choose a complementary distribution $Q$ to $K$ so that $T M=Q \oplus K$ and fix $p \in f^{-1}(q), q \in N$. If $Y, Z \in Q_{p}$, then at $q$ we have

$$
\operatorname{Ric}^{D}\left(f_{*} Y, f_{*} Z\right)=(n-1) \gamma(Y, Z)+2 d \sigma(Z, Y)+(1-n) \Sigma(Y, Z) ;
$$

since $\operatorname{Ric}^{D}$ is symmetric, we get $\left.d \sigma\right|_{Q \times Q}=0$ and $\operatorname{Ric}^{D}\left(f_{*} Y, f_{*} Z\right)=(n-1)[\gamma(Y, Z)-$ $\Sigma(Y, Z)]$. If we insert this in (1.4), we find that the projective curvature tensor $W_{N}$ vanishes.

Proposition 1.2. If $\mathcal{P}_{M}$ is a complete flat projective structure on $M$ and $f: M \rightarrow N$ is a projective submersion, then $\operatorname{dim} M=\operatorname{dim} N$ and $f$ is a covering map.

Proof: Let $(\widetilde{M}, p)$ be the universal covering manifold of $M$, with the induced projective structure. Since $\mathcal{P}_{M}$ is complete and flat, $\widetilde{M}$ is projectively diffeomorphic to the $m$-sphere $S^{m}$ and $f \circ p: S^{m} \rightarrow N$ is a projective submersion. The structure $\mathcal{P}_{S^{m}}$ is induced by the canonical metric connection on $S^{m}$, which has a symmetric parallel, positive definite Ricci tensor; let $\sigma$ be the 1 -form corresponding to $f \circ p$. We note that we can make use of formula (1.4), since the structure $\mathcal{P}_{S^{m}}$ is flat. If we choose $X=Z \in K$ and $Y \notin K$, we obtain that $\gamma(X, X)=\Sigma(X, X)$. We now consider the geodesic $\rho: \mathbf{R} \rightarrow S^{m}$ starting from a point $q \in S^{m}$ and with initial vector $X_{q}$ : since the fibres of $f \circ p$ are totally geodesic, the geodesic $\rho$ lies entirely in the fibre through the point $q$. Moreover

$$
\gamma(\dot{\rho}, \dot{\rho})=\left(\nabla_{\dot{\rho}} \sigma\right) \dot{\rho}-(\sigma(\dot{\rho}))^{2} .
$$

Since $\gamma$ is parallel, if $X_{q}$ is not zero, then $\gamma\left(\dot{\rho}_{t}, \dot{\rho}_{t}\right)=a>0$ for all $t \in R$, for some constant $a \in \mathbf{R}$. So if we put $\lambda(t)=\sigma\left(\dot{p}_{t}\right)$, we have

$$
\frac{d}{d t} \lambda(t)-\lambda(t)^{2}=a ;
$$

hence

$$
\arctan \frac{\lambda(t)}{\sqrt{a}}-\arctan \frac{\lambda(0)}{\sqrt{a}}=\sqrt{a} t \quad \forall t \in \mathbf{R},
$$

and this is impossible since arctan is a bounded function. So $K=\{0\}$ and $f \circ p$ is a local diffeomorphism. Since $S^{m}$ is compact, we have that $f \circ p$, hence $f$, is a covering map.

We now want to generalise the previous result. In $[1]_{1}$ Blumenthal has considered Cartan-submersions between manifolds endowed with projective structures; these are 
submersions covered by a bundle map of the corresponding bundles of 2-frames inducing the projective structures. The definition we are going to give yields an analogous condition for the projective mappings we are considering.

We denote by $W_{M}, W_{N}$ the projective curvature tensors of $\mathcal{P}_{M}$ and $\mathcal{P}_{N}$ respectively and put the following:

Definition 1.2: A projective map $f:\left(M, \mathcal{P}_{M}\right) \rightarrow\left(N, \mathcal{P}_{N}\right)$ is said to be $W$ projective if

$$
f_{*} W_{M}(X, Y) Z=W_{N}\left(f_{*} X, f_{*} Y\right) f_{*} Z
$$

for all vector fields $X, Y, Z$ on $M$.

We note that every local projective diffeomorphism is $W$-projective. According to the notation used before, we now state some consequences of the $W$-projectiveness. If we assume that $\nabla$ and $D$ have symmetric Ricci tensors and put $\gamma=R_{i c} D /(m-1)$ and $\delta=\operatorname{Ric}^{\nabla} /(n-1)$, we have that (1.5) together with (1.3) imply

$$
\begin{aligned}
W_{N}\left(f_{*} X, f_{*} Y\right) f_{*} Z=f_{*} & W_{M}(X, Y) Z+\left[\gamma(Y, Z)-\Sigma(Y, Z)-\delta\left(f_{*} Y, f_{*} Z\right)\right] f_{*} X \\
& +\left[\gamma(X, Z)-\Sigma(X, Z)-\delta\left(f_{*} X, f_{*} Z\right)\right] f_{*} Y+2 d \sigma(X, Y) f_{*} Z .
\end{aligned}
$$

So if we suppose that $\operatorname{dim} N \geq 3$ we get that $d \sigma=0$ and

$$
\delta\left(f_{*} X, f_{*} Y\right)=\gamma(X, Y)-\Sigma(X, Y) .
$$

Equation (1.7) means that $f$ maps geodesics into geodesics preserving the class of projective parameters, see [3]. We can now generalise Proposition 1.2 to a larger class of manifolds: in [4] Gray considered $\mathcal{A}$-manifolds, namely Riemannian manifolds $(M, g)$ whose Ricci tensor $S$ satisfies

$$
\left(\nabla_{X} S\right)(X, X)=0
$$

for every vector field $X$. Among others he showed that there exist $\mathcal{A}$-manifolds which are not Einstein and he exhibited a structure of $\mathcal{A}$-manifold on the quotient space $O(4) / O(2)$ which is not projectively flat. In general we call an $\mathcal{A}$-manifold a $C^{\infty}$ manifold endowed with a torsionfree affine connection whose Ricci tensor is symmetric and satisfies condition (1.8).

THEOREM 1.1. Let $M$ be a complete $\mathcal{A}$-manifold with positive definite Ricci tensor. If $f: M \rightarrow\left(N, \mathcal{P}_{N}\right)$ is a $W$-projective submersion, then $f$ is a covering map, provided $\operatorname{dim} N \geq 3$.

Proof: We first prove that $f$ is a local diffeomorphism. We note that if $\{\rho\}_{t \in R}$ is a geodesic for $(M, g)$, then the function $\gamma\left(\dot{\rho}_{t}, \dot{\rho}_{t}\right)$ is a positive constant by condition 
(1.8) and our hypotheses on the Ricci tensor. Now using equation (1.7) we can apply the same argument as in the proof of Proposition 1.2 to get that $f_{*}$ is everywhere oneto-one. In order to prove our assertion, we are going to apply a result of Blumenthal ([2]) stating that a local projective diffeomorphism of a complete projectively connected manifold onto an arbitrary projectively connected manifold is a covering map. So we have to show that $(M, g)$ is projectively complete, that is every projective parameter along any geodesic can be extended over the whole real line. Let $\rho: \mathbf{R} \rightarrow M$ be any geodesic with affine parameter $s$ : thanks to condition (1.8), the Schwarz equation defining a projective parameter $p$ along $\rho$ is (see [3])

$$
\{p, s\}=\frac{1}{2} k^{2}
$$

where $k^{2} / 2=2 \gamma\left(\dot{\rho}_{0}, \dot{\rho}_{0}\right)$. The solutions of this equation are of the form

$$
p(s)=a \tan \frac{k s+b}{2}+c \quad(a \neq 0)
$$

for some real constants $a, b, c$, so that every projective parameter can be extended on $\mathbf{R}$.

\section{REFERENCES}

[1] R.A. Blumenthal, 'Cartan submersions and Cartan foliations', Mlinois J. Math. 31 (1987), 327-343.

[2] R.A. Blumenthal, 'Local isomorphisms of projective and conformal structures', Geom. Dedicata 16 (1984), 73-78.

[3] L.P. Eisenhart, Non-Riemannian Geometry: Colloq. Publ. 8 (Amer. Math. Soc., 1927).

[4] A. Gray, 'Einstein-like manifolds which are not Einstein', Geom. Dedicata 7 (1978), 259-280.

[5] Z. Har'el, 'Projective mappings and distorsion theorems', J. Diff. Geometry 15 (1980), 97-106.

Scuola Normale Superiore

P.zza Cavalieri

1-56100 Pisa

Italy 